\title{
A new large-scale meta-epidemiological study on bias in randomized trials using routinely collected risk-of-bias assessments by cochrane reviewers: results from the robes study
}

\author{
Jelena Savovic ${ }^{1 *}$, Rebecca Turner ${ }^{2}$, David Mawdsley ${ }^{1}$, Julian Higgins' ${ }^{1}$, Jonathan Sterne ${ }^{1}$ \\ From 3rd International Clinical Trials Methodology Conference \\ Glasgow, UK. 16-17 November 2015
}

\section{Introduction}

Empirical evidence suggests that certain aspects of trial design may lead to biased intervention effect estimates. We examined the influence of risk-of-bias judgements from Cochrane reviews for sequence generation, allocation concealment, blinding and incomplete data on intervention effect estimates in a large collection of meta-analyses (MAs).

\section{Methods}

We selected MAs with dichotomous outcomes and $>4$ included trials from intervention reviews with fully completed risk-of-bias tool, published in issue 4, 2011 of the Cochrane Library. We classified outcome measures as mortality, other objective or subjective, and estimated the effect of risk-of-bias domain judgements on average bias (ratios of odds ratios [ROR] with 95\% credible intervals [Cr-I]) using Bayesian hierarchical models.

\section{Results}

Among 2815 trials in 256 meta-analyses, intervention effect estimates were on average exaggerated in trials with high or unclear risk-of-bias (versus low) for random sequence generation (ROR 0.91 [95\% Cr-I 0.86, 0.98]), for allocation concealment (ROR 0.92 [95\% Cr-I 0.86-0.98]) and for blinding (ROR 0.87 [95\% Cr-I 0.80, 0.93]). Unlike our previous study, we did not observe consistently different bias or between-trial heterogeneity in bias in MAs with subjective outcomes compared to mortality. Results from analyses of the influences of incomplete data were inconclusive.

University of Bristol, Bristol, UK

Full list of author information is available at the end of the article

\section{Limitations}

Possible inconsistency in criteria for risk-of-bias judgments applied by individual reviewers is a likely limitation of routinely collected bias assessments.

\section{Conclusions}

Inadequate randomization or lack of blinding may lead to exaggeration of intervention effect estimates in trials, but it is unclear if this effect differs by outcome type.

\section{Authors' details}

${ }^{1}$ University of Bristol, Bristol, UK. ${ }^{2}$ MRC Biostatistics Unit, Cambridge, UK.

Published: 16 November 2015

doi:10.1186/1745-6215-16-S2-P168

Cite this article as: Savovic et al:: A new large-scale meta-

epidemiological study on bias in randomized trials using routinely

collected risk-of-bias assessments by cochrane reviewers: results from the robes study. Trials 2015 16(Suppl 2):P168.

Submit your next manuscript to BioMed Central and take full advantage of:

- Convenient online submission

- Thorough peer review

- No space constraints or color figure charges

- Immediate publication on acceptance

- Inclusion in PubMed, CAS, Scopus and Google Scholar

- Research which is freely available for redistribution 